\title{
The Analysis and Amendment of Security System in 3G
}

\author{
Min ZHAO, Ling SHI \\ Computer Department, East China University of Science and Technology, Shanghai, China
}

\begin{abstract}
This paper analyses security mechanism and authentication, key agreement protocol of $3 \mathrm{G}$ system amply, puts forward a amelioration scheme. This scheme assures security communications without credible VLR. This paper analyses ameliorative protocol and resolves the security communications problem between MS and HLR.
\end{abstract}

Keywords: 3G Security, 3G Security Structure, 3G authentication and key agreement protocol Security arithmetic

\section{G 系統安全體制分析與協議改進}

\author{
趙 敏, 史 令 \\ 華東理工大學 計算機系 200237
}

\begin{abstract}
摘 要: 本文對 $3 \mathrm{G}$ 系統安全體制和認證、密錀分配協議進行了詳細分析, 提出了對協議的改進方案, 從而保 證有不完全可信 VLR 參與的保密通信，並對改進方案進行了分析，解決了 MS 到 HLR 之間的通信安全問題。
\end{abstract}

關鍵詞: $3 \mathrm{G}$ 安全, $3 \mathrm{G}$ 安全體制, $3 \mathrm{G}$ 認證與密錀協商, 安全算法

\section{1. 引 言}

在第三代移動通信系統中, 除了傳統的話音業務 外，電子商務、電子貿易、網路服務等新型業務將成 為 $3 \mathrm{G}$ 的重要業務內容。目前, $2 \mathrm{G}$ 網路已經投入了大 量資金和設備, 廢棄 $2 \mathrm{G}$ 網路在一種新的安全體制之 上開發 $3 \mathrm{G}$ 網路是不現實的作法。移動通信中 $3 \mathrm{G}$ 網路 與 $2 \mathrm{G}$ 網路的共存是目前移動通信向 $3 \mathrm{G}$ 過渡必然要經 歷的階段。因此, $3 \mathrm{G}$ 網路要繼承 $2 \mathrm{G}$ 網路, 無法改變 建立在對稱密碼安全體制之上的 $2 G$ 安全體制。從 1996 年起, 歐洲電信標準學會著手開發 3G 移動系 統, 並逐漸由 $3 \mathrm{GPP}$ 取代, 其目的是制定一個全球統 一的移動應用規範。 $3 \mathrm{G}$ 安全的設計宗旨是在 $2 \mathrm{G}$ 安全 的基礎上有所提高, 以提供新的安全性能與服務。因 此, 在移動通信系統中, 資訊的加密仍將採用對稱密 碼體制, 為實現資訊的加密傳輸, 通信雙方必須首先 進行身份認證, 協商會話密錀 $[1]$ 。在現有 $2 \mathrm{G}$ 網路中
引入 $3 \mathrm{G}$ 接入設備和網路實體時,要在儘量不改變原有 $2 \mathrm{G}$ 網路實體的基礎上,實現與現有 $2 \mathrm{G}$ 網路的交互, 使移動網路能為雙模式手持設備用戶提供通過使用 SIM 卡或 USIM 卡在 $2 G$ 和 $3 G$ 網路的接入和服務 $[2]$

$3 \mathrm{G}$ 安全體制不是端到端的安全機制, 給密級較高 的應用帶來困難。比如, 如果 VLR 在一個不被信任的 網路域中, 由於在 $3 \mathrm{G}$ 系統中它可以獲取通信所需的 CK 和 IK 的, 這樣對 VLR 而言, 就没有什麼秘密可 言。

\subsection{G 安全體制}

$3 \mathrm{G}$ 的安全目標是: 保證由用戶產生的或與用戶相 關的資訊能夠得到充分保護, 防止被誤用或盜用; 保 證由服務網路或歸屬環境提供的資源和業務能夠得到 充分保護，防止被誤用或盜用; 保證標準化的安全特 徵至少應有一個可以在世界範圍的基礎上輸出的加密 
演算法; 保證安全特徵被充分的標準化, 以確保世界 範圍內互操作與不同的服務網路之間的漫遊; 保證提 供給用戶和業務供應者的保護級別比現代固定和移動 網路提供的高; 保證 3GPP 安全特徵、機制和實現能 被擴展和加強[3]。

$3 \mathrm{G}$ 安全體制的總體結構分為 3 個層面和 5 個部 分:

(1) 網路接入安全: 提供安全接入 $3 \mathrm{G}$ 服務網的機 制，並抵禦無線鏈路攻擊。這一部分功能包括：用戶 身份保密、認證和密錀分配、資料加密和完整性等。 認證過程也融合了加密、完整性保護等措施。

（2）網路域安全: 保證網內信令的安全傳送並抵禦 對有線網路的攻擊。

（3）用戶域安全: 主要保證對移動台的安全接入， 包括用戶與智慧卡間認證、智慧卡與終端的認證及其 鏈路的保護。

（4）應用域安全: 使用戶域與服務提供商的應用程 式間能夠安全的交換資訊。

（5）安全特性的可視性及可配置能力: 主要指用戶 能獲知安全特性是否在使用以及服務提供商提供的服 務是否需要以安全服務為基礎[4]。

加密和完整性保護是實現安全通信的核心, 認證 與密錀分配是實現安全通信的重要保證。3G 系統的安 全體制是建立在 $2 \mathrm{G}$ 的基礎上, GSM 及其他 $2 \mathrm{G}$ 系統 中已被證明是必須和䅼健的安全元素將繼續被採用, $3 \mathrm{G}$ 還將改進 $2 \mathrm{G}$ 中的安全弱點, 最終提供全新的安全 性能和業務。3G 相關安全協議很多, 本文針只對其中 的核心部分, 認證與密錀分配協議進行分析與改進。

\section{3G 認證與密錀分配協議分析與改進}

$3 \mathrm{G}$ 安全系統定義了一些安全演算法, 下面只介紹 本文所用到的安全演算法:

$\mathrm{f} 1=>\mathrm{MAC}$ : 產生消息認證碼;

$\mathrm{f} 2=>\mathrm{XRES}$ : 用於消息認證中計算期望回應值;

$\mathrm{f3}=>\mathrm{CK}$ : 產生加密密錀;

$\mathrm{f} 4=>\mathrm{IK}$ : 產生完整性密錀;

$\mathrm{f5}=>\mathrm{AK}$ ：產生匿名密錀；

$3 \mathrm{G}$ 認證與密錀分配由 $\mathrm{fl} \sim \mathrm{f5}$ 實現。

$3 \mathrm{G}$ 規範中 UMTS 鑒權過程是建立在每個用戶唯 一的用戶錀匙 $\mathrm{K}$ 的基礎上的, $\mathrm{K}$ 存儲在 USIM 和用戶 環境歸屬 $\mathrm{AuC}$ 中。MS：移動站; AKA：認證與密錀 分配; UE: 用戶終端; HLR: 歸屬位置寄存器; VLR:
訪問位置寄存器; HE：本地環境；AV：認證向量, $\mathrm{AV}=\mathrm{RAND}\|\mathrm{XRES}\| \mathrm{CK}\|\mathrm{IK}\| \mathrm{AUTH} \quad ; \quad \mathrm{XRES}=\mathrm{f} 2(\mathrm{~K}$ ， RAND) ; $C K=\mathrm{f}(\mathrm{K}, \mathrm{RAND})$; $\mathrm{IK}=\mathrm{f} 4(\mathrm{~K}, \mathrm{RAND})$; $\mathrm{AUTH}=\mathrm{SQN} \oplus \mathrm{AK}\|\mathrm{MODE}\| \mathrm{MAC} ; \mathrm{MODE}$ : 移動性管 理標識; MAC $=\mathrm{fl}(\mathrm{K}, \mathrm{SQN}\|\mathrm{RAND}\| \mathrm{MODE})$ 。

\section{1 協議過程分析}

認證協定分為以下幾個步驟:

(1) MS $\rightarrow$ VLR: IMSI $\mid$ HLR;

(2) VLR $\rightarrow$ HLR: IMSI;

(3) HLR $\rightarrow$ VLE: 認證向量 AV;

(4) VLR $\rightarrow$ MS: RAND $\|$ AUTH;

(5) MS $\rightarrow$ VLR: RES

首先 VLR 收到 MS 的註冊請求, MS 發送用戶自 己的 IMSI 和 HLR 資訊給 VLR;

VLR 接收到該請求後, 將 IMSI 轉發給 MS 的 HLR;

HLR 接到 IMSI 後產生 SQN, RAND, 按照上述 公式計算認證向量 $\mathrm{AV}$ ，併發送給 VLR;

VLR 接收到 AV 後, 將解析到的 RAND 和 AUTH 轉發給 MS;

MS 接收到認證請求回應後, 計算 $\mathrm{XMAC}=\mathrm{f} 1(\mathrm{~K}$, SQN |RAND ||MODE), 比較是否等於 MAC, 如果不 同, 則向 VLR 發送拒綛認證消息並放棄該過程。接下 來檢測 SQN 是否在合理的範圍內, 如果不在合理範圍 內，就向 VLR 發送“同步失敗”消息，放棄該過程。如 果上面檢測都通過了, MS 計算 RES、CK 和 IK, 並 將 RES 發送給 VLR;

VLR 接收到 RES 後, 與 XRES 比較, 如果 RES 與 XRES 相同, 認證成功, 否則認證失敗。協定流程 如圖 1 所示。

\section{2 安全性分析}

前面已經提到 MS 與 HLR 之間共用一個密錀 K, 該密錀的安全是 $3 \mathrm{G}$ 安全體制的基本保障。因為 $3 \mathrm{G}$ 安 全體制建立在對稱密碼體制基礎上, 所以對稱密錀的 安全顯得尤為重要。

（1）認證安全分析：我們已經知道，3G 安全認證 是雙向認證, MS 需要認證 HLR, VLR 需要認證 MS。

VLR 接收到來自 HLR 的認證向量中包含了期望 MS 產生的應答 $X R E S=f 2(K ， R A N D) 。$ 若 $M S$ 是合法 用戶，在接收到 VLR 返回的 RAND 後, 只有合法的 


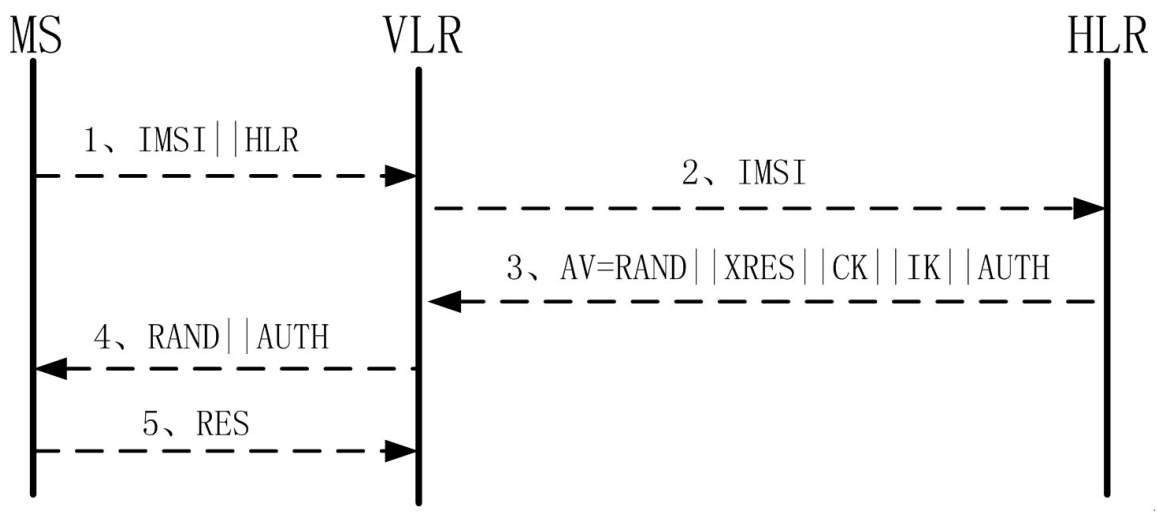

圖 1. 認證與密錀分配過程

Figure 1. Authentication \& key distribution process

$M S$ 才能計算出 $R E S=f 2(K, R A N D)$, 且 $R E S=X R E S$ 。 因為 $\mathrm{K}$ 由 MS 和其歸屬的 HLR 所掌握, 所以別人無 法計算得出 RES, 這樣, 就實現了 VLR 對 MS 的認證

MS 對 HLR 的認證是通過消息認證碼 MAC 來實 現的 MS 接收到 VLR 轉發的來自 HLR 的 MAC $=\mathrm{fl}(\mathrm{K}$, SQN $\|$ RAND $\|$ MODE), 計算 XMAC $=f 1(K, S Q N \| R A N D$ $\| \mathrm{MODE})$, 在保證 $\mathrm{SQN}$ 的正確性前提下, $\mathrm{MAC}=\mathrm{XM}$ $\mathrm{AC}$, 則認證成功。因為對稱密錀 $\mathrm{K}$ 在 HLR 也掌握, 隨 意只有合法的 HLR 才能計算出與 MS 計算得到相同的 MAC 值，這樣就實現了 MS 對 VLR 和 HLR 的認證。

（2）加密和完整性密錀每次會話都更新

MS 與 VLR 之間每次通信均採用不同的密錀 $\mathrm{CK}$ 與 $I K_{\circ}$ 。由於每次通信前的認證選擇了不同的認證向 量, 保證每次通信採用的 CK 和 IK 是採用不同的亂數 得到的。而每次使用的消息認證碼 MAC 是由不斷遞 增的序列號 SQN 作為其輸入參數之一, 保證了認證消 息的新鮮性, 從而確保密錀的新鮮性, 但在一次會話 建立後直到該會話結束 CK 與 IK 不會改變。

(3) 資料完整性

通過在消息中包含哈希值來進行完整性驗證。在 認證和密錀分配過程中, 通信雙方協商了完整性密錀 IK，通過 IK 可以保證資料没有被修改。

(4) MS 與 VLR 之間的密錀分配安全性

VLR 接收到來自 HLR 的認證向量中包含了加密 密錀 $\mathrm{CK}$ 與完整性密錀 IK, 合法用戶在收到正確的亂 數 RAND 後, 能正確產生 $\mathrm{CK}=\mathrm{f} 3(\mathrm{~K}, \mathrm{RAND}), \mathrm{CK}$ 與 IK 未在無線介面中傳輸, 確保了密錀的安全性。也就 是說, CK 與 IK 在 HLR 和 MS 端分別產生, 由於有 共用密錀 $\mathrm{K}$, 所以雙方產生的 $\mathrm{CK}$ 和 IK 是一致的。

\section{3 協議改進}

從上面分析可以看出, 目前 $3 \mathrm{G}$ 通過在 MS 和 HLR 共用密錀，達到 MS 與 VLR 之間加密通信。也就是說 MS 可以用與 VLR 之間協商的 CK 和 IK 進行加密通 信，但無法從 $3 \mathrm{G}$ 安全協議本身保證從 VLR 和 HLR 之間的安全通信，VLR 和 HLR 之間的安全通信是通 過網路域的保密措施來保證的, $3 \mathrm{G}$ 協議本身並未對次 進行定義。

$3 \mathrm{G}$ 的認證與密錀分配協議在假定 HLR 與 VLR之 間安全的前提下，實現了用戶終端對網路 HLR/VLE 的認證和網路對用戶的認證, 同時也實現了在 UE 與 VLR 之間的密錀分配。從協議上看， $3 \mathrm{G}$ 認證與密錀 分配協議不是一種端到端的安全協議, 在 VLR 和 HLR 之間是通過網路域安全來保證的。

如果第三方在 VLR 和 HLR 之間對資訊進行艄 取，就可以獲取 HLR 傳送給 VLR 的認證向量 AV, 從而可以獲取 CK 和 $\mathrm{IK}$ 。這樣, MS 的加密資料對入 侵者而言就没有秘密可言。

針對上述分析，有兩種方案進行改進：

（1）對可信的 VLR 而言，增加 HLR 與 VLR 之間 的共用密錀 $\mathrm{K} 2$;

通過增加 HLR 與 VLR 共用密錀, 實現 HLR 和 VLR 之間重要資訊的加密傳輸, 保證了傳輸資訊的機 密性，使第三方無法獲取認證向量 $\mathrm{AV}$, 保證通信的 安全[5]。

本方法解決了在 VLR 和 HLR 之間的安全傳輸問 題，雖然這可以用網路域的安全方案給予解决，但本 方案從 $3 \mathrm{G}$ 協議本身入手, 解決了第三方窲取通信資 
料的問題。

（2）對不完全可信的 VLR 而言，利用 MS 與 HLR 之間共用的密錀 $\mathrm{K}$ 進行加密通信, VLR 只作轉發工 作，這適用於密級較高的應用。

從圖 1 可以看出, 在 VLR 和 HLR 之間部分傳輸 資料對不可信 VLR 是敏感的，比如：認證向量 $\mathrm{AV}$ 。 如果不希望 VLR 見到敏感資料, 可以利用共用密錀 K 進行處理。假設對稱加密演算法為 $\mathrm{E}(\bullet)$; 對稱解密演 算法為 $\mathrm{D}(\bullet)$;

(1) MS $\rightarrow$ VLR: IMSI $\mid H L R$;

(2) VLR $\rightarrow$ HLR: IMSI;

(3) HLR $\rightarrow$ VLE: $\mathrm{E}(\mathrm{K}, \mathrm{AV}) \| \mathrm{XRES}$;

(4) VLR $\rightarrow$ MS: $\mathrm{E}(\mathrm{K}, \mathrm{AV})$;

(5) MS $\rightarrow$ VLR: RES

注: $A V=R A N D \| A U T H$

在改進協議中, HLR 利用與 MS 共用的密錀 $\mathrm{K}$ 對 AV 加密處理, 並計算 XRES, 把 $\mathrm{E}(\mathrm{K}, \mathrm{AV})|| \mathrm{XRES}$ 傳送 給 VLR。由於 VLR 無法掌握密錀 $\mathrm{K}$, 也就無法掌握 認證向量 $A V$ 。VLR 把 $E(K, A V)$ 轉發給 $M S$, 由於 $M S$ 掌握密錀 $K$, 所以可以得到認證向量 $A V$ 。從 $A V$ 中可以獲取 RAND 和 AUTH, MS 計算得到 CK 和 IK 用於加密通信, 計算 $R E S=f 2(K, R A N D)$ 返回給 $V L R 。$ VLR 比較 XRES 與 RES, 如果相等就認證成功。此過 程見圖 2。

此方法解決了如果在 VLR 不可信的基礎上, 如何 保證端到端的資料加密通信。第三方或不可信的 VLR 無法獲取敏感通信資料。

安全性分析:
加密通信過程中, 用密錀 $\mathrm{CK}$ 進行加密, 完整性 驗證用密錀 $\mathrm{IK}$ 。CK 和 IK 由 RAND 和共用密錀 $\mathrm{K}$ 產 生, 而 $\mathrm{K}$ 只有 $\mathrm{MS}$ 和 HLR 掌握, 所以只有 MS 和 HLR 能夠產生正確的 CK 和 IK, 從而保證通信安全。

假設, 建立會話後進行後續通信的資料為 Data, 對稱加密演算法為 $\mathrm{E}(\bullet)$; 對稱解密演算法為 $\mathrm{D}(\bullet)$; MS 把 Data 進行對稱加密結果 $\mathrm{E}(\mathrm{CK}$, Data) ||HLR 發 送給 VLR，VLR 轉發給 HLR。HLR 利用 CK 進行解 密得到 Data。這樣, 在通信線路中傳輸的為密文, 從 而確保安全。

假設某個 VLR 不可信, 由於它無法計算得到加密 密錀 $\mathrm{CK}$ ，從而無法得到加密通信資料 $\mathrm{E}(\mathrm{CK}$ ，Data) 的明文 Data，資料就可以安全通過 VLR 到達 HLR 了。

假設竊聽者在通信線路上截取通信資料，與 VLR 一樣，它也無法計算得到加密密錀 $\mathrm{CK}$ ，也無法獲取 通信資料的明文。

該協定繼續沿用了 3GPP 標準中該協議的安全特 性：對 MS 和 HLR/VLR 進行雙向認證; 在認證過程 中進行密錀分配和協商; 資料完整性驗證等。

此協定中，只是把 VLR 作為通信資料轉發站來 看，VLR 和任何想截獲通信資料的第三方都無法得到 通信資料明文，從而有效的防止了第三方竊取和 VLR 不可信的問題，但這會增加一些計算量，對通信效率 有所影響。對密級較高的通信，損失一些速度而保證 安全也是可取的。

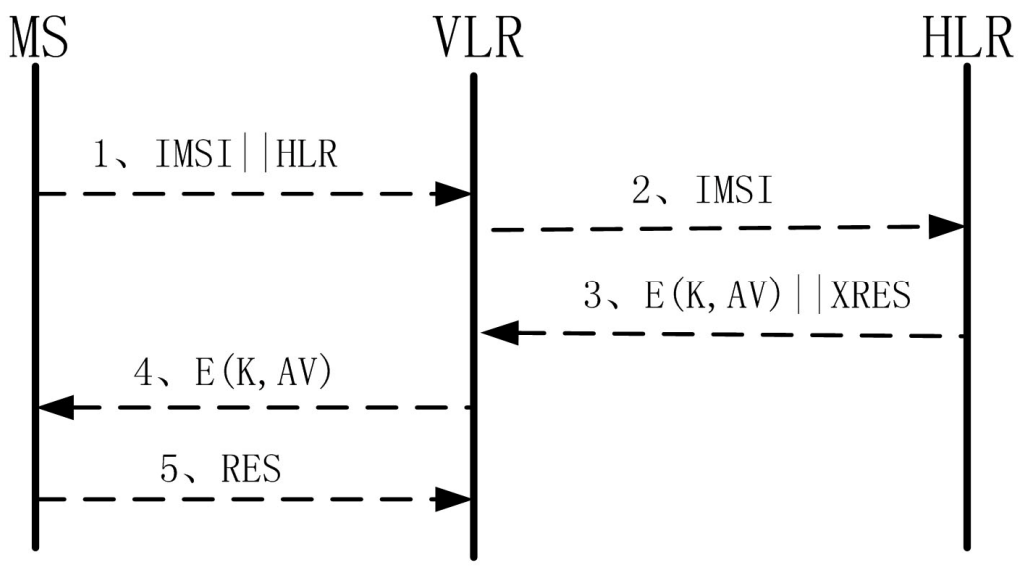

圖 2. 改進的認證與密錀分配協定

Figure 2. Improved authentication \& key distribution agreements 


\section{4. 結論}

目前 $3 \mathrm{G}$ 相關的協議和標準正在不斷完善過程 中, 本文從理論角度提出瞭解决在不完全可信 VLR 參與情況下安全通信的問題。目前 $3 G$ 系統中 HLR 和 VLR 之間網路傳輸的安全特性定義在網路域的安全 協定中, 本文從 $3 \mathrm{G}$ 協議本身出發, 提出了建立在對 稱密碼機制基礎之上結合 $3 \mathrm{GPP}$ 相關協議的安全認證 與密錀分配協議的新思路。

該安全協議有如下特點:

繼承 $3 \mathrm{G}$ 認證與密錀分配協議的優點, 不損害原 有協議安全性;

從協議自身增強 VLR 與 HLR 之間的通信安全 性; 能夠抵抗中間人攻擊。

\section{REFERENCES}

[1] Zhu li-qi, Huang ben-xong. improvement and formal analysis of
$3 \mathrm{G}$ authentication and key distribution protocol. Electronics Engineers, 05/2004(In Chinese) (朱裏奇, 黃本雄. $3 \mathrm{G}$ 認證和密 錀分配協議的形式化分析與改進. 電子工程師, 5/2004).

[2] Liu zi-long, lu Zheng-xin, huang zhai-lu. 2G and 3G mobile network system security and user authentication. Telecommunications technology, 02/2002(In Chinese)(劉子龍, 盧正新, 黃載祿. $2 \mathrm{G}$ 與 $3 \mathrm{G}$ 移動網系統安全性及用戶鑒權. 電 訊技術, 2/2002).

[3] Li xiang, 3G Security Architecture. Telecommunications Technology, 10/2002(In Chinese)(李翔. 3G 的安全體系結構. 電信技術, 10/2002).

[4] Lin de-jing, lin bai-gang, lin de-qing. research and analysis of 3G system-wide network security. ZTE Technology. 02/2003 (In Chinese)(林德敬, 林柏岡, 林德清. $3 \mathrm{G}$ 系統全網安全體制 的探討與分析. 中興通訊技術, 2/2003).

[5] Liu dong-shu, wei bao-dian, wang xin-mei. improvement of the $3 \mathrm{G}$ authentication and key distribution protocol. Journal of Communication, 05/2002(In Chinese)(劉東蘇, 韋寶典, 王新梅. 改進的 $3 \mathrm{G}$ 認證與密錀分配協定 通信學報. 5/2002). 